\title{
Inhibitory Effects of a Herbal Ointment against Pseudomonas aeruginosa
}

\author{
Maryam Meskini (PhD Candidate) \\ Department of Microbiology and \\ Applied Microbiology Research \\ Center, Systems Biology and \\ Poisonings Institute, Baqiyatallah \\ University of Medical Sciences, \\ Tehran, Iran \\ Azad Khaledi (PhD) \\ Department of Microbiology, Kashan \\ University of Medical Science, \\ Kashan, Iran \\ Davoud Esmaeili (PhD) \\ Department of Microbiology and \\ Applied Microbiology Research \\ Center, Systems Biology and \\ Poisonings Institute, Baqiyatallah \\ University of Medical Sciences, \\ Tehran, Iran, and Applied \\ Virology Research Center, \\ Baqiyatallah University of Medical \\ Sciences \\ Corresponding author: Davoud \\ Esmaeili \\ Email: esm114@gmail.com \\ Tel: +989124395884 \\ Address: Iran, and Applied \\ Virology Research Center, \\ Baqiyatallah University of Medical \\ Sciences \\ Received: 04 Sep 2017 \\ Revised: 02 Dec 2017 \\ Accepted: 04 Dec 2017

\section{(c) (1) (3)}

This work is licensed under a Creative Commons Attribution 4.0 License.
ABSTRACT

Background and Objectives: Pseudomonas aeruginosa is a gram negative opportunistic pathogen and an important cause of wound infections and nosocomial infections. The purpose of this study was to study inhibitory effects of a new ointment prepared from medicinal plants against $P$. aeruginosa isolates.

Methods: In this study, an ointment called Z0LSH was prepared from mixing alcoholic extracts of Satureja khuzestaniea, Zataria multiflora, Mentha mozaffariani Jamzad, honey and polyurethane. Minimal inhibitory concentration of Z0LSH and its compositions alone or combined was determined using the disk diffusion method.

Results: S. khuzestaniea, Z. multiflora and Mentha mozaffariani Jamzad had inhibitory effects against $P$. aeruginosa. The ZoLSH ointment had greater antibacterial effects than the any of its compositions used solely or combined. The diameter of inhibition zone had a direct relationship with the concentration of the extracts. Moreover, the antibacterial effect of the ZOLSH ointment was identical to that of polymyxine B (300 $\mu \mathrm{g})$.

Conclusion: We demonstrated that the Z0LSH ointment has inhibitory effects against $P$. aerugionosa. The inhibition zone diameter is directly correlated with the concentration of the extracts. Our results suggest that the ointment could be useful for treatment of burn wounds and skin infections.

Keywords: Pseudomonas aeruginosa, Z0LSH, Burns, Satureja khuzestaniea, Zataria multiflora, Mentha Mozaffariani Jamzad, Honey, Polyurethane, Ointment. 


\section{INTRODUCTION}

Pseudomonas aeruginosa is the third most common cause of nosocomial infections and the second most important cause of wound infections (1). Emergence of multi drug resistant (MDR) and extensively drug resistant (EDR) strains of $P$. aeruginosa as well as the increased rate of resistant infections in burn patients highlights the importance of infection control.

$P$. aeruginosa species have various virulence factors such as pili, exo-enzyme A, exotoxins, etc. (2). Infection caused by this bacterium is believed to be responsible for $75 \%$ of all mortalities in burn patients $(3,4)$. Given the high prevalence of $\beta$-lactamase production by $P$. aeruginosa and the high rate of antibiotic resistance, researchers have become more interested in seeking medicinal herbs with antibacterial properties that are safe and without side effects of chemical drugs (5). One of the most important health complications is the infections secondary to burn wounds and war wounds. Dau and et al. reported that $7 \%$ of war-related injuries are burn wounds (6). Satureja khuzestanica is an herbaceous, aromatic and perennial herb from the family Lamiaceae. Thyme and Satureja are thought to have antimicrobial activities due to presence of phenolic compounds, thymol and carvacrol (7, 8). Zataria multiflora and Oregano (Origanum vulgare $L$.) are also medicinal plants from the family Lamiaceae (9). It has been suggested that S. khuzestanica, Z. multiflora and Mentha mozaffariani Jamzad may have antibacterial activity, particularly against $P$. aeruginosa (10). In this study, we aimed to assess the antibacterial activity of $S$. khuzestaniea, Mentha mozaffariani Jamzad and $Z$. multiflora, honey and polyurethane against $P$. aeruginosa isolates.

\section{MATERIALS AND METHODS}

Arial parts of S. khuzestaniea, Mentha mozaffariani Jamzad and Z. multiflora were collected from Ahvaz (southwestern Iran), Kermanshah (western Iran) and Shiraz (southcentral Iran), respectively. The collected plants were fragmented to small pieces $(200 \mathrm{~g})$ and dried. Alcoholic extractions of the plants were prepared at Barij Essence Kashan, Iran.

$P$. aeruginosa $\mathrm{PAO} 1$ was used as the reference strain. Susceptibility of the isolates to different antibiotics was assessed by disc diffusion method according to the Clinical Laboratory Standard Institute guidelines (CLSI 2016). The following antibiotic disks were used in the susceptibility testing: polymyxine B (300 $\mathrm{U} / \mathrm{IE})$, tobramycin $(10 \mu \mathrm{g})$, gentamycin (10 $\mu \mathrm{g})$, imipenem $(10 \mu \mathrm{g})$ and meropenem (10 $\mu \mathrm{g})$. The antimicrobial activity of the plant extracts, honey and polyurethane was also determined by the disk diffusion method. To prepare the ZOUSH ointment (mixture of the plant extract, honey and polyurethane), one gram of each plant extract was dissolved in 3 $\mathrm{ml}$ of dimethyl sulfoxide (DMSO), and one gram of honey and one gram of polyurethane were each dissolved in $3 \mathrm{ml}$ of water. $P$. aeruginosa PAOI $\left(1.5 \times 10^{8} \mathrm{CFU} / \mathrm{ml}\right)$ were spread on Muller-Hinton agar plates. Subsequently, disks containing different concentrations of the ointment were placed on the medium. Later, the plates were incubated at $37{ }^{\circ} \mathrm{C}$ for 24 hours. The diameter of inhibition zone was measured in $\mathrm{mm}$. A disk previously immersed in DMSO was used as the negative control. The experiments were repeated three times.

Minimal inhibitory concentration (MIC) was determined by broth macro dilution assay according to the National Committee for Clinical Laboratory Standard guidelines. First, a bacterial stock solution with a turbidity equivalent to $0.5 \mathrm{McF}$ arland turbidity standard $\left(1.5 \times 10^{8} \mathrm{CFU} / \mathrm{ml}\right)$ was prepared. The plate was incubated at $37{ }^{\circ} \mathrm{C}$ for 24 hours. A tube without the ZOUSH stock was used as negative control. A medium without turbidity was considered as resistant. Lowest concentration at which the growth of bacteria was inhibited was determined as the MIC. $P$. aeruginosa was identified by morphological assessment, catalase test, oxidase test, oxidation-reduction test, Pseudomonas agar base medium and pigment formation.

\section{RESULTS}

All tubes were re-cultured on Muller Hinton agar to determine the MIC values using the broth macrodilution technique. Table 1 shows the results of MIC values for $P$. aeruginosa PAOI using broth macrodilution and microdilution methods. The results showed that the ZOUSH ointment had greater antibacterial effects than the any of its compositions used solely or combined. 
Table 1 -MIC values for the ZOUSH ointment and its compositions

\begin{tabular}{cc}
\hline Agent & MIC $(\boldsymbol{\mu g} / \mathbf{m L})$ \\
\hline S. khuzestaniea & 0.079 \\
Z. multiflora & 0.079 \\
Mentha mozaffariani Jamzad & 0.158 \\
Honey & 0.079 \\
S. khuzestaniea + Z. multiflora & 0.059 \\
S. khuzestaniea + Mentha & 0.079 \\
mozaffariani Jamzad & \\
Z. multiflora + Mentha & 0.079 \\
mozaffariani Jamzad & \\
ZOUSH oinment & 0.039 \\
\hline
\end{tabular}

Susceptibility of $P$. aeruginosa PAO1 to the antibiotics was assessed using the Kirby-Bauer method. The results showed that $P$. aeruginosa PAO1 was sensitive to gentamicin, imipenem, meropenem, polymyxine $\mathrm{B}$ and tobramycin (Table 2).

The susceptibility of $P$. aeruginosa PAO1 to different concentrations of the ZOUSH and its compostions alone or combined was evaluated. Based on the results, the growth inhibition zone for $20 \mu \mathrm{g}$ of single extracts (S. khuzestaniea, Z. multiflora, Mentha mozaffariani Jamzad), double extracts (Z.multiflora and S. khuzestaniea, Mentha mozaffariani Jamzad and S. khuzestaniea, Mentha mozaffariani Jamzad and $Z$. multiflora), triple extract (Mentha mozaffariani Jamzad, S. khuzestaniea and Z. multiflora) and the ZOUSH ointment was almost identical to that of the polymyxin B $(300 \mu \mathrm{g})$.

In addition, the inhibition zone diameter had a direct relationship with the extract's concentration (Table 3).

Table 3- Diameter of inhibition zone for different concentrations of the ZOUSH ointment and the extracts alone and combined

\begin{tabular}{ccccc}
\hline Agent & $10 \mu \mathrm{g}$ & $13 \mu \mathrm{g}$ & $17 \mu \mathrm{g}$ & $20 \mu \mathrm{g}$ \\
\hline S. khuzestaniea & $9 \mathrm{~mm}$ & $10 \mathrm{~mm}$ & $12 \mathrm{~mm}$ & $16 \mathrm{~mm}$ \\
Z. multiflora & $8 \mathrm{~mm}$ & $9 \mathrm{~mm}$ & $12 \mathrm{~mm}$ & $14 \mathrm{~mm}$ \\
Mentha mozaffariani Jamzad & $9 \mathrm{~mm}$ & $11 \mathrm{~mm}$ & $12 \mathrm{~mm}$ & $15 \mathrm{~mm}$ \\
S. khuzestaniea + Z. multiflora & $10 \mathrm{~mm}$ & $12 \mathrm{~mm}$ & $14 \mathrm{~mm}$ & $15 \mathrm{~mm}$ \\
S. khuzestaniea + Mentha mozaffariani Jamzad & $12 \mathrm{~mm}$ & $14 \mathrm{~mm}$ & $15 \mathrm{~mm}$ & $16 \mathrm{~mm}$ \\
Z. multiflora + Mentha mozaffariani Jamzad & $10 \mathrm{~mm}$ & $13 \mathrm{~mm}$ & $14 \mathrm{~mm}$ & $16 \mathrm{~mm}$ \\
S. khuzestaniea + Z. multiflora + Mentha & $11 \mathrm{~mm}$ & $13 \mathrm{~mm}$ & $14 \mathrm{~mm}$ & $17 \mathrm{~mm}$ \\
mozaffariani Jamzad & $12 \mathrm{~mm}$ & $14 \mathrm{~mm}$ & $15 \mathrm{~mm}$ & $18 \mathrm{~mm}$ \\
ZOUSH ointment & & & &
\end{tabular}

\section{DISCUSSION}

$P$. aeruginosa is an important opportunistic, gram-negative bacillus and a main cause of mortality among immunocompromised patients $(11,12)$. Burn injuries are one of the most serious medical conditions, which affect the patients both physically and psychologically. Despite the recent scientific and therapeutic advances, burns still remain as a major public health problem around the world, especially in developing countries, such as Iran $(13,14)$. Moreover, the increasing trend of resistant $P$. aeruginosa infection of burn wounds has raised some concerns, and signified the need for implementation of effective infection control program for burn patients. However, prevention of $P$. aeruginosa spread in hospital settings is challenging because of the bacterium's intrinsic and acquired resistance to numerous antibiotics. Additionally, the emergence of MDR and XDR strains has become a worldwide health problem. Traditional medicine plays an important role in the treatment and prevention of infections caused by these bacteria (15). Carvacrol is a major component of the $S$. khuzestaniea extract, which inhibits ATPase activity and increases bacterial cell membrane 
permeability, thus facilitating the access of antibacterial agents (16). In 2015, Farzaneh et al. showed that carvacrol, $\gamma$-terpinene and $\mathrm{p}$ cymene are the main components of $S$. khuzestaniea. They also demonstrated the antifungal properties of various species of Satureja and the antimicrobial activity of ZOUSH ointment compounds (17). Similar to our study, Esmaeili et al. reported the inhibitory effect of $S$. khuzestaniea against $P$. aeruginosa (18).

In a study conducted by Moghim et al., the antifungal effect of $Z$. multiflora and Nigella Sativa was proven against Candida albicans (19). Kavoosi et al. investigated the effect of Z. multiflora on Salmonella typhimurium, Escherichia coli, Staphylococcus aureus, Staphylococcus epidermidis, Aspergillus niger and $C$. albicans. They claimed that the thymol and carvacrol content of Z. multiflora has a direct relationship with its antioxidant, antibacterial, antifungal and anti-tumor

\section{REFERENCES}

1. Van Delden C, Iglewski BH. Cell-to-cell signaling and Pseudomonas aeruginosa infections. Emerg Infect Dis. 1998; 4(4): 551-60. DOI:10.3201/eid0404.980405.

2. Cobb LM, Mychaleckyj JC, Wozniak DJ, LópezBoado YS. Pseudomonas aeruginosa flagellin and alginate elicit very distinct gene expression patterns in airway epithelial cells: implications for cystic fibrosis disease. J Immunol. 2004; 173(9): 5659-5670.

3. Sadikot RT, Blackwell TS, Christman JW, Prince AS. Pathogen-host interactions in Pseudomonas aeruginosa pneumonia. Am J Respir Crit Care Med 2005; 171(11):1209-1223.

4. Holder IA. Pseudomonas immunothera〉yy: A historical overview. Vaccine. 2004; 22(7):831-839. DOI:10.1016/j.vaccine.2003.11.028.

5. Sadikot RT, Blackwell TS, Christman JW, Prince AS. Pathogen-host interactions in Pseudomonas aeruginosa pneumonia. Am J Respir Crit Care Med. 2005; 171(11): 1209-23.

6. Wurtz R, Karajovic M, Dacumos E, Jovanovic B, Hanumadass M. Nosocomial infection in a burn intensive care unit. Burns. 1995; 21(3): 181-184.

7. Hosseini SMJ, Shoaee NN, Azad Khaledi AC, Daymad F, Esmaeili D. Evaluate the Relationship Between Class 1 Integrons and Drug Resistance Genes in Clinical Isolates of Pseudomonas aeruginosa. Open Microbiol J. 2016; 10: 188-196.

8. Soltani K, Zand R, Mirghasemi A. Epidemiology and mortality of burns inTehran, Iran. Burns. 1998; 24(4): 325-328.

9. Esmaeili D, Moradi F, Foladi AI, Bahador A. The Study of Inhibitory Effects of Satureja khuzestanicaEssence against Exos Gene of MDR Pseudomonas aeruginosa by RT-PCR Technique. Pharmaceut Reg Affairs. 2015; 4: 3. properties (20). Our results also confirm the antibacterial properties of $Z$. multiflora.

\section{CONCLUSION}

We demonstrated that the ZOUSH ointment has inhibitory effects against $P$. aerugionosa. The inhibition zone diameter is directly correlated with the concentration of the extracts. Our results suggest that the ointment could be useful for treatment of burn wounds and skin infections.

\section{ACKNOWLEDGEMENTS}

The authors would like to thank the staff of the University of Baqiyatallah Medical Sciences and Imam Khomeini hospital for assisting us in specimen collection.

\section{CONFLICT OF INTEREST}

The authors declare that there is no conflict of interest.

10. Meskini M, Ghorbanalizadegan M, Esmaeili D. Importance of Herbal Ointment for Pseudomonas aeruginosa infection in burn patients. Int J Med Invest. 2015; 4(2): 257-261.

11. Lotfi N, Shokohi T. A review on fungal infection in burn patients, diagnosis and treatment. J Mazandaran Univ Med Sci. 2014; 23(108): 151-165.

12. Yousefi E, Rafienia M, Fazeli H, Kasai MZ. In-Vitro Effects of Copper Nanoparticles on Common Bacterial Strains Implicated in Nosocomial Infections. Journal of Isfahan Medical School. 2013; 31(240):830-842.

13. Alaghebandan R, Rossignol AM, Rastegar Lari A. Pediatric Burn Injuries in Tehran, Iran. Burns. 2001; 27(2): 115-118.

14. Darvishpour A, Lotfi M, Salehi F, Aghazadeh A, Hasani A, Ali N. Comparing Dressing With Silver Sulfadiazine and Nitrofurazone to the Pediatrice Ward in Tabriz. J of Guilan Univer of Med Science. 2006; 15(60): 42-46.

15. Yavarpour V, Zarabi M, Esmaeili D, Mohamadnejad J. In vitro Evaluation of Antibacterial Efficacy of Natural Honeys in Comparison with Antibiotics on Pseudomonas aeruginosa. Iran J Med Microbiol 2014, 8(1): 34-37.

16. Dorman H, Deans S. Antimicrobial agents from plants: antibacterial activity of plant volatile oils. J Appl Microbiol. 2000; 88(2): 308-316.

17. Farzaneh M, Kiani H, Sharifi R, Reisi M, Hadian J. Chemical composition and antifungal effects of three species of Satureja (S. hortensis, S. spicigera, and S. khuzistanica) essential oils on the main pathogens of strawberry fruit. Postharvest Biology and Technology. 2015; 109: 145-151. 
18. Abbasi A, Bahador A, Esmaeili D, Mahbubi A, Amiri M, Amiri M. The Study of Inhibitory Effects of Satureja khuzestanica against MDR Isolates of Pseudomonas aeruginosa. International $\mathrm{J}$ of Current Microbiology and Applied Sciences. 2014; 2(3):614-618. 19. Moghim H, Taghipoor S, Shahinfard N, Kheiri S,
Panahi R. Antifungal effects of Zataria multifora and Nigella sativa extracts against Candida albicans. J HerbMed Pharmacol. 2015; 4(4):138-141.

20. Kavoosi G, Rabiei F. Zataria multiflora: chemical and biological diversity in the essential oil. Journal of Essential Oil Research. 2015; 27(5): 428-436. 